\title{
Prevalence and Antimicrobial Susceptibility Pattern of Urinary Tract Infection among Pregnant Women Attending Hargeisa Group Hospital, Hargeisa, Somaliland
}

\author{
Abdikhaliq Hussein Ali \\ Hawassa University College of Medicine and Health Science \\ Dawit Yihdego Reda \\ Hawassa University College of Medicine and Health Science \\ Moges Desta Ormago ( $\square$ moges22@gmail.com) \\ Hawassa University College of Medicine and Health Science
}

\section{Research Article}

Keywords: study, susceptibility, hargeisa, participants, bacteria

Posted Date: October 13th, 2021

DOI: https://doi.org/10.21203/rs.3.rs-957306/v1

License: () (1) This work is licensed under a Creative Commons Attribution 4.0 International License. Read Full License

Version of Record: A version of this preprint was published at Scientific Reports on January 26th, 2022. See the published version at https://doi.org/10.1038/s41598-022-05452-z. 


\section{Abstract}

The aim of this study was to determine the prevalence, antimicrobial susceptibility pattern and associated factors of urinary tract infection (UTI) among pregnant women attending Hargeisa Group Hospital ( $\mathrm{HGH})$, Hargeisa, Somaliland. A cross-sectional study was conducted at HGH, Hargeisa, Somaliland and participants were selected by systematic random sampling technique. Clean catch midstream urine samples were collected from 422 participants and cultured and antimicrobial susceptibility pattern was determined for the isolates. Univariable and multivariable logistic regression analyses were utilized to identify the independent risk factors for UTI

The prevalence of UTI was $16.4 \%$ (95\% Cl: 13.3-19.9). The predominant bacteria isolate was E. coli (43.5\%). Gram negative bacteria were resistant to ampicillin (96\%) and tetracycline (71.4\%) and Gram-positive bacteria were also resistant to ampicillin (90\%), tetracycline (55\%). Multidrug resistance was observed in $89.9 \%$ of bacterial isolated. No formal education participants were 3.18 times, previous history of catheterization had 3.22 times and previous history of UTI had 3.73 times more likely to develop UTI than their counterparts respectively. Culture and susceptibility test is vital for appropriate management of UTI in the study area.

\section{Introduction}

Urinary tract infection (UTI) is one of the highest frequent problem caused by some bacteria in a pregnant woman, which can lead to cause the significant complications for both mother and fetus ${ }^{1}$. The prevalence of the infection is higher among pregnant women than non-pregnant women and it is a major health problem reported among $20 \%$ of the pregnant women and a common cause of admission in obstetrical wards ${ }^{2}$.

If the infection is left untreated, it results in low birth weight fetus, intrauterine growth retardation, preterm labor and premature babies, intrauterine fetal death, and increased prenatal mortality and morbidity as well as maternal complications including anemia, preeclampsia, renal failure, septicemia, and adult respiratory syndrome ${ }^{3}$.

In Hargeisa Group Hospital, Hargeisa, Somaliland, routine culture and antimicrobial susceptibility testing of UTI are not performed and the treatment is on an empirical basis. This may promote the overuse of antibiotics and the development of resistant microbial strains. And there was no published information on the prevalence of UTI and antimicrobial susceptibility pattern in Somaliland. Therefore, this study was carried out to determine the prevalence, antimicrobial susceptibility pattern and associated factors of urinary tract infection among pregnant women attending antenatal care at $\mathrm{HGH}$, Hargeisa, Somaliland.

\section{Method}

Cross-sectional study was conducted at antenatal care (ANC) in HGH, Hargeisa, Somaliland from May to October 2020. Being pregnant and having a follow up in the ANC clinic of HGH were included in the study. Pregnant women who received antibiotics within two weeks before ANC follow up were excluded. A structure questionnaire was used by trained nurses for the collection of Clinical and Socio-demographic data.

Ten milliliters of clean catch midstream urine sample were collected in a wide mouthed sterile container from each study participant. The collected urine sample was labeled and delivered to the hospital laboratory within one hour ${ }^{4}$.

The collected urine samples were processed and using a calibrated loop $(0.001 \mathrm{ml})$ urine specimens were inoculated in to blood agar and MacConkey agar plates. After overnight incubation at $37^{\circ} \mathrm{C}$ for $24-48$ hours' colonies were counted to check significant growth. Colony counts of bacterial growth of $>10^{5} / \mathrm{ml}$ of urine were significant ${ }^{5,6}$. All positive cultures with significance bacteriuria were then identified at species level by their colony characteristics, gram-staining reaction and by the pattern of biochemical profiles using the standard microbiological technique. The enterobacteriaceae were identified by indole production, $\mathrm{H} 2 \mathrm{~S}$ production in KIA agar, citrate utilization, motility test, urease test, oxidase and carbohydrate utilization tests. The gram positive bacteria were identified using catalase and coagulase tests ${ }^{4}$.

Kirby-Bauer disc diffusion method was used for the antibiotic susceptibility test. Three to five pure colonies were transferred into a tube containing $4-5 \mathrm{~mL}$ nutrient broth and mixed gently and then incubated at $35-37^{\circ} \mathrm{C}$ for $2-6 \mathrm{hrs}$. The turbidity of the suspension was compared with $\mathrm{McF}$ arland 0.5 tubes to standardize the inoculums size ${ }^{7}$.

By using a sterile cotton swab dipping it into the suspension evenly over the entire surface of Mueller-Hinton agar (MHA) (Oxide Ltd, Hampshire, UK). The inoculated plates were left at room temperature to dry for 3-15 minutes. The following commercially available antibiotic discs were used with their respective

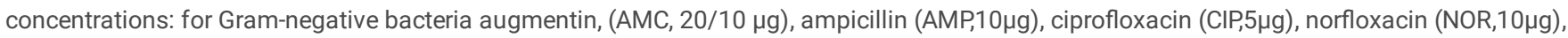

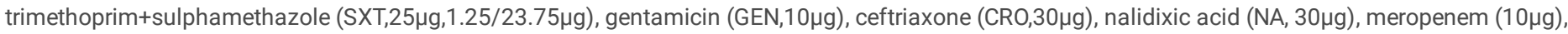

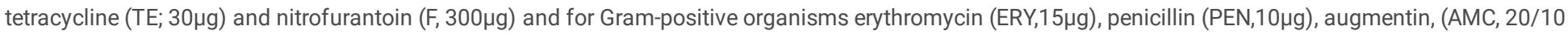

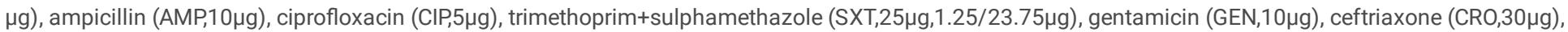

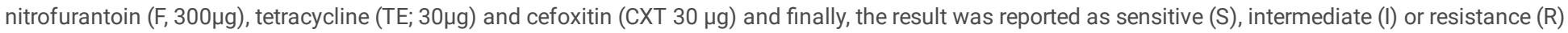
by measuring the diameter of zone of inhibition or hemolysis ${ }^{8}$.

All filled questionnaires for this study was checked visually, coded and entered into excel and then exported to SPSS version25 software (SPSS Inc., Chicago, IL, USA) for analysis. Bivariate logistic regression was used to determine predictors of culture confirmed UTI. For those variables, which $p$-value $<0.25$ in the bivariate, the analysis was further entered into the multivariable logistic regression model ${ }^{9}$. Associations between dependent and independent variables were assessed and its strength was described using odds ratios at $95 \%$ confidence intervals. A statistically significant association considered as $p$-value $<0.05$. 
Ethical approval was obtained from the institutional review board of Hawassa University, College of medicine and health sciences (Ref No: IRB/204/12). Informed consent was obtained from all participants. All methods were carried out in accordance with relevant guidelines and regulations.

\section{Results}

A total of 422 pregnant women were participated during the study period. In this study the mean age of study participants was 30.9 ( \pm 5.6 SD) years within the age range of 18-44. Majority of the study participants $125(29.6 \%)$ was $31-35$ and $119(28.2 \%)$ was $26-30$ years old, $407(96.4 \%)$ was married and $398(94.3 \%)$ was urban dwellers. Among study participants, 79(18.7\%) had no formal education, 243(57.6\%) was housewives, and 191(45.3\%) was earned \$101-200 per month (Table1).

Table1. Sociodemographic characteristics of pregnant women attended antenatal care at HGH, Hargeisa, Somaliland, 2020 ( $n=422$ ).

\begin{tabular}{|c|c|c|c|}
\hline Variables & & Frequency & Percent (\%) \\
\hline \multirow[t]{6}{*}{ Age (in years) } & $<19$ & 17 & 4.0 \\
\hline & $20-25$ & 88 & 20.9 \\
\hline & $26-30$ & 119 & 28.2 \\
\hline & $31-35$ & 125 & 29.6 \\
\hline & $36-40$ & 40 & 9.5 \\
\hline & $41-45$ & 33 & 7.8 \\
\hline \multirow[t]{2}{*}{ Residence } & Urban & 398 & 94.3 \\
\hline & Rural & 24 & 5.7 \\
\hline \multirow[t]{4}{*}{ Marital status } & Widowed & 8 & 1.9 \\
\hline & Married & 407 & 96.4 \\
\hline & Divorced & 2 & 0.5 \\
\hline & Separated & 5 & 1.2 \\
\hline \multirow[t]{4}{*}{ Educational status } & No formal education & 79 & 18.7 \\
\hline & Primary education (1-8) & 185 & 43.8 \\
\hline & Secondary education(9-12) & 112 & 26.5 \\
\hline & Higher education (>12) & 46 & 10.9 \\
\hline \multirow[t]{4}{*}{ Family Income } & $<\$ 100$ & 77 & 18.2 \\
\hline & $\$ 101-200$ & 191 & 45.3 \\
\hline & $\$ 201-300$ & 116 & 27.5 \\
\hline & $<\$ 300$ & 38 & 9.0 \\
\hline \multirow[t]{4}{*}{ Occupational status } & House wife & 243 & 57.6 \\
\hline & Merchant & 92 & 21.8 \\
\hline & Governmental employee & 78 & 18.5 \\
\hline & Student & 9 & 2.1 \\
\hline
\end{tabular}

In this study 198 (46.9\%), 137 (32.5\%), and 87(20.6\%) were in the 3rd, 2nd and 1st trimester of pregnancy respectively. Among the study participants, 366 $(86.7 \%)$ had multigravida, 56 (13.3\%) were primigravida, 28 (6.6\%) had prior use of indwelling catheter, $10(2.4 \%)$ had diabetes mellitus. Among the study participants, history of abortion, History of obstetric and gynecologic surgery, History of premature labor and history of UTI, were found in $17(4 \%), 9(2.1 \%)$ $6(1.4 \%)$ and $52(12.3 \%)$ of study participants respectively (Table2).

Table2. Obstetrics and clinical characteristics of pregnant women attended antenatal care at HGH, Hargeisa, Somaliland, 2020 ( $\mathrm{n}=422$ ). 


\begin{tabular}{|c|c|c|c|}
\hline Variables & & equency(n) & Percent (\%) \\
\hline \multirow[t]{3}{*}{ Gestational Period } & First trimester & 87 & 20.6 \\
\hline & Second trimester & 137 & 32.5 \\
\hline & Third trimester & 198 & 46.9 \\
\hline \multirow[t]{2}{*}{ Gravida } & Primigravida & 56 & 13.3 \\
\hline & Multigravida & 366 & 86.7 \\
\hline \multirow[t]{2}{*}{ History of Catheterization } & No & 394 & 93.4 \\
\hline & Yes & 28 & 6.6 \\
\hline \multirow[t]{2}{*}{ History of diabetes mellitus } & No & 412 & 97.6 \\
\hline & Yes & 10 & 2.4 \\
\hline \multirow[t]{2}{*}{ History of abortion } & No & 405 & 96.0 \\
\hline & Yes & 17 & 4.0 \\
\hline \multirow[t]{2}{*}{ History of obstetric and gynecologic surgery } & No & 413 & 97.9 \\
\hline & Yes & 9 & 2.1 \\
\hline \multirow[t]{2}{*}{ History of premature labor } & No & 416 & 98.6 \\
\hline & Yes & 6 & 1.4 \\
\hline \multirow[t]{2}{*}{ History of previous UTI } & No & 370 & 87.7 \\
\hline & Yes & 52 & 12.3 \\
\hline
\end{tabular}

Overall prevalence of UTI was $16.4 \%$ (95\% Cl: $13.3-19.9)$ of which $40(9.5 \%)$ was symptomatic UTI and $29(6.9 \%)$ was asymptomatic UTI. Of 69 positive cases, eight different types of bacteria were identified. The majority of the isolates belong to the Gram negative bacteria $49(71 \%)$. Among the isolates the predominant bacteria were E. coli 30(43.5\%), followed by Coagulase negative staphylococcus (CONs) 11(16\%), S. aureus 9(13\%), K. pneumonia 6(8.7\%), Pseudomonas aeruginosa 5(7.2\%), Proteus mirabilis 4(5.8\%), Citrobacter spp 3(4.4\%) and M. morganii 1(1.5\%) (Table3).

Table3. Bacterial profile isolated from urine culture of symptomatic and asymptomatic pregnant women with UTI attended antenatal care at HGH, Hargeisa, Somaliland, $2020(n=69)$.

\begin{tabular}{|c|c|c|c|}
\hline Bacterial isolates & Asymptomatic UTI(n=29) & Symptomatic UTI $(n=40)$ & Total $(n=69)$ \\
\hline \multicolumn{4}{|l|}{ Gram negative } \\
\hline E. coli & $12(17.4 \%)$ & $18(26.1 \%)$ & $30(43.5 \%)$ \\
\hline K. pneumoniae & $1(1.5 \%)$ & $5(7.2 \%)$ & $6(8.7 \%)$ \\
\hline$P$. aeruginosa & $2(2.9 \%)$ & $3(4.3 \%)$ & $5(7.2 \%)$ \\
\hline P. mirabilis & $2(2.9 \%)$ & $2(2.9 \%)$ & $4(5.8 \%)$ \\
\hline Citrobacter spp. & $1(1.5 \%)$ & $2(2.9 \%)$ & $3(4.4 \%)$ \\
\hline M. morganii & $1(1.5 \%)$ & $0(0)$ & $1(1.5 \%)$ \\
\hline Sub total & $19(27.5 \%)$ & $30(43.5 \%)$ & $49(71 \%)$ \\
\hline \multicolumn{4}{|l|}{ Gram positive } \\
\hline CoNS & $6(8.7 \%)$ & $5(7.2 \%)$ & $11(16 \%)$ \\
\hline S. aureus & $4(5.8 \%)$ & $5(7.2 \%)$ & $9(13 \%)$ \\
\hline Sub total & $10(14.5 \%)$ & $10(14.5)$ & $20(29)$ \\
\hline Total & $29(42 \%)$ & $40(58 \%)$ & $69(100 \%)$ \\
\hline
\end{tabular}

Abbreviations: CONS, coagulase-negative staphylococci; UTI, urinary tract infection.

Gram negative bacteria were resistant to ampicillin (96\%), tetracycline (71.4\%), trimethoprim-sulfamethoxazole (57.1\%), amoxicillin clavulanic acid (55.1\%) and nalidixic acid (51\%) and high rate of sensitive were also observed to meropenem (95.9\%), ceftriaxone (79.6\%), norfloxacin (77.5\%), gentamicin (75.5\%), nitrofurantoin (75.5\%) and ciprofloxacin (71.4\%). With regard to specific isolated Gram negative bacteria, Escherichia coli were highly resistant to ampicillin (93.3\%), tetracycline (73.3\%), trimethoprim-sulfamethoxazole (60\%), nalidixic acid (53.3\%) and amoxicillin clavulanic acid (46.7\%) but sensitive to meropenem (96.7\%), norfloxacin (90\%), ceftriaxone (83.3\%), nitrofurantoin (80\%), gentamicin (73.3\%) and ciprofloxacin (66.7\%). 
All K. pneumonia isolates showed resistant to ampicillin, and $66.7 \%$ were resistant to norfloxacin and amoxicillin clavulanic acid each and $50 \%$ were resistant to tetracycline, nalidixic acid and trimethoprim-sulfamethoxazole each but $K$. pneumonia was sensitive to meropenem (100\%), gentamycin (83.3\%) and 66.7\% were sensitive to ceftriaxone, ciprofloxacin and nitrofurantoin each.

P. aeruginosa were resistant to ampicillin (80\%), tetracycline (57.1\%), trimethoprim- sulfamethoxazole (57.1\%) while highly sensitive were observed to meopenem, gentamicin, norfloxacin and ciprofloxacin to $80 \%$ for each and $60 \%$ were sensitive to ceftriaxone and nitrofurantoin each. Proteus mirabilis were resistant to ampicillin (100\%) and 75\% to each of tetracycline and amoxicillin clavulanic acid while $100 \%$ sensitive to meropenem, gentamicin and ceftriaxone each and $75 \%$ sensitive to ciprofloxacin and norfloxacin each. Citrobacter spp were highly resistant to ampicillin (100\%) and $66.7 \%$ were resistant to tetracycline, nalidixic acid, trimethoprim-sulfamethoxazole and amoxicillin clavulanic acid each but Citrobacter spp were highly sensitive to meropenem, ciprofloxacin and nitrofurantoin and $66.7 \%$ were sensitive to gentamicin, norfloxacin and ceftriaxone each.

M. morganii were resistant to ampicillin, tetracycline, nalidixic acid, trimethoprim-sulfamethoxazole, norfloxacin and amoxicillin clavulanic acid but all $M$. morganii were sensitive to meropenem, ceftriaxone, ciprofloxacin and nitrofurantoin (Table4).

Table4. Antimicrobial Susceptibility Pattern of Gram-negative Bacteria Isolated from pregnant women with UTI attended antenatal care at HGH, Hargeisa, Somaliland, $2020(n=49)$

\begin{tabular}{|c|c|c|c|c|c|c|c|c|c|c|c|c|}
\hline \multirow[t]{2}{*}{ Isolates } & \multirow[t]{2}{*}{ Pattern } & \multicolumn{11}{|c|}{ Antibiotics (\%) } \\
\hline & & AMP & CIP & CRO & AMC & SXT & NOR & GEN & NA & $\mathrm{F}$ & MER & TTC \\
\hline \multirow{3}{*}{$\begin{array}{l}\text { E. coli }(\mathrm{N}= \\
30)\end{array}$} & $\mathrm{S}$ & $1(3.3)$ & $20(66.7)$ & 25(83.3) & 14(46.7) & $9(30)$ & $27(90)$ & 22(73.3) & 13(43.3) & $24(80)$ & $29(96.7)$ & $4(13.3)$ \\
\hline & 1 & $1(3.3)$ & $2(6.7)$ & & $2(6.7)$ & $3(10)$ & $1(3.3)$ & $2(6.7)$ & $1(3.3)$ & $2(6.7)$ & & $4(13.3)$ \\
\hline & $\mathrm{R}$ & 28(93.3) & $8(26.7)$ & $5(16.37)$ & $14(46.7)$ & $18(60)$ & $2(6.7)$ & $6(20)$ & 16(53.3) & $4(13.3)$ & 1(3.3) & $22(73.3)$ \\
\hline \multirow{3}{*}{$\begin{array}{l}\text { K. } \\
\text { pneumonia } \\
(\mathrm{N}=6)\end{array}$} & $S$ & & $4(66.7)$ & $4(66.7)$ & 1(16.7) & $1(16.7)$ & 2(33.3) & $5(83.3)$ & $3(50)$ & $4(66.7)$ & $6(100)$ & 1(16.7) \\
\hline & 1 & & & 2(33.3) & $1(16.7)$ & 2(33.3) & & & & & & 2(33.3) \\
\hline & $\mathrm{R}$ & $6(100)$ & $2(33.3)$ & & $4(66.7)$ & $3(50)$ & $4(66.7)$ & $1(16.7)$ & $3(50)$ & $2(33.3)$ & & $3(50)$ \\
\hline \multirow{3}{*}{$\begin{array}{l}P . \\
\text { aeruginosa } \\
(\mathrm{N}=5)\end{array}$} & $S$ & & $4(80)$ & $3(60)$ & $2(40)$ & $1(20)$ & $4(80)$ & $4(80)$ & $2(40)$ & $3(60)$ & $4(80)$ & $1(20)$ \\
\hline & I & & & & & $1(20)$ & $1(20)$ & & $1(20)$ & $1(20)$ & $1(20)$ & \\
\hline & $\mathrm{R}$ & $5(100)$ & $1(20)$ & $2(40)$ & $3(60)$ & $3(60)$ & & $1(20)$ & $2(40)$ & $1(20)$ & & $4(80)$ \\
\hline \multirow{3}{*}{$\begin{array}{l}\text { P. mirabilis } \\
(\mathrm{N}=4)\end{array}$} & $S$ & & $3(75)$ & $4(100)$ & $1(25)$ & $2(50)$ & $3(75)$ & $4(100)$ & $2(50)$ & $2(50)$ & $4(100)$ & $1(25)$ \\
\hline & I & & & & & $1(25)$ & & & $1(25)$ & & & \\
\hline & $\mathrm{R}$ & $4(100)$ & $1(25)$ & & $3(75)$ & $1(25)$ & $1(25)$ & & $1(25)$ & $2(50)$ & & $3(75)$ \\
\hline \multirow{3}{*}{$\begin{array}{l}\text { Citrobacter } \\
\text { spp. }(\mathrm{N}=3)\end{array}$} & S & & $3(100)$ & $2(66.7)$ & 1(33.3) & 1(33.3) & $2(66.7)$ & $2(66.7)$ & 1(33.3) & $3(100)$ & $3(100)$ & \\
\hline & 1 & & & & & & 1(33.3) & & & & & $1(33.3)$ \\
\hline & $\mathrm{R}$ & $3(100)$ & & $1(33.3)$ & $2(66.7)$ & $2(66.7)$ & & 1(33.3) & $2(66.7)$ & & & $2(66.7)$ \\
\hline \multirow{3}{*}{$\begin{array}{l}\text { M. morganii } \\
(\mathrm{N}=1)\end{array}$} & S & & $1(100)$ & $1(100)$ & & & & & & $1(100)$ & $1(100)$ & \\
\hline & 1 & & & & & & & $1(100)$ & & & & \\
\hline & $\mathrm{R}$ & $1(100)$ & & & $1(100)$ & $1(100)$ & $1(100)$ & & $1(100)$ & & & $1(100)$ \\
\hline \multirow[t]{3}{*}{ Total $(\mathrm{N}=49)$} & S & $1(2)$ & $35(71.4)$ & $39(79.6)$ & 19(38.8) & $14(28.6)$ & $38(77.5)$ & $37(75.5)$ & $21(42.9)$ & $37(75.5)$ & $47(95.9)$ & $7(14.2)$ \\
\hline & I & $1(2)$ & $2(4.1)$ & $2(4.1)$ & $3(6.1)$ & $7(14.3)$ & $3(6.1)$ & $3(6.1)$ & $3(6.1)$ & $3(6.1)$ & $1(2)$ & $7(14.2)$ \\
\hline & $\mathrm{R}$ & $47(96)$ & $12(24.5)$ & $8(16.3)$ & $27(55.1)$ & $28(57.1)$ & $8(16.3)$ & $9(18.4)$ & $25(51)$ & $9(18.3)$ & $1(2)$ & $35(71.4)$ \\
\hline
\end{tabular}

Abbreviations: AMP: ampicillin, CIP: ciprofloxacin, CRO: ceftriaxone, AMC: amoxicillin clavulanic acid, SXT: trimethoprim-sulfamethoxazole, NOR: norfloxacin, GEN: gentamicin, NA: nalidixic acid, F: nitrofurantoin, MER: meropenem, TTC: tetracycline S, I, R, sensitive, intermediate, resistant

The Gram-positive bacteria were resistant to ampicillin (90\%), tetracycline (55\%), trimethoprim-sulfamethoxazole (50\%), and amoxicillin clavulanic acid (50\%) while sensitive to erythromycin (85\%), cefoxitin (85\%), ceftriaxone (75\%), nitrofurantoin (75\%), gentamicin (70\%) and ciprofloxacin (85\%).

Coagulase negative staphylococci (CoNs) were highly resistant to ampicillin (81.8\%), tetracycline (54.5\%), and amoxicillin clavulanic acid (45.4\%) but sensitive to erythromycin (81.8\%), cefoxitin (81.8\%), ceftriaxone (72.7\%), nitrofurantoin (72.7\%) and gentamicin (72.7\%), ciprofloxacin (63.6\%) and trimethoprim-sulfamethoxazole (54.5\%)

S. aureus were highly resistant to ampicillin (100\%) and 55.6\% were resistant to tetracycline, trimethoprim- sulfamethoxazole and amoxicillin clavulanic acid each while sensitive to erythromycin (88.9\%), cefoxitin (88.9\%), ceftriaxone (77.8\%) and nitrofurantoin (77.8\%), ciprofloxacin (66.7\%) and gentamicin (66.7\%) 
(Table5).

Table5. Antimicrobial Susceptibility Pattern of Gram-positive bacteria isolated from pregnant women with UTI attended antenatal care at HGH, Hargeisa, Somaliland, $2020(n=20)$

\begin{tabular}{|c|c|c|c|c|c|c|c|c|c|c|c|c|}
\hline Isolates & Pattern & \multicolumn{11}{|c|}{ Antibiotics (\%) } \\
\hline \multirow[t]{3}{*}{ CONs $(\mathrm{N}=11)$} & S & $1(9.1)$ & $7(63.6)$ & $8(72.7)$ & $5(45.4)$ & $6(54.5)$ & $9(81.8)$ & $8(72.7)$ & $5(45.4)$ & $9(81.8)$ & $8(72.7)$ & $3(27.3)$ \\
\hline & I & $1(9.1)$ & $2(18.2)$ & & $1(9.1)$ & & & $1(9.1)$ & $2(18.2)$ & $1(9.1)$ & $1(9.1)$ & $2(18.2)$ \\
\hline & $\mathrm{R}$ & $9(81.8)$ & $2(18.2)$ & $3(27.3)$ & $5(45.4)$ & $5(45.4)$ & $2(18.2)$ & $2(18.2)$ & $4(36.4)$ & $1(9.1)$ & $2(18.2)$ & $6(54.5)$ \\
\hline \multirow{2}{*}{$\begin{array}{l}\text { S. aureus } \\
(\mathrm{N}=9)\end{array}$} & I & & $1(11.1)$ & $1(11.1)$ & $3(33.3)$ & & & $2(22.2)$ & $1(11.1)$ & & & 1(11.1) \\
\hline & $\mathrm{R}$ & $9(100)$ & $2(22.2)$ & $1(11.1)$ & $5(55.6)$ & $5(55.6)$ & $1(11.1)$ & $1(11.1)$ & $4(44.4)$ & $1(11.1)$ & $2(22.2)$ & $5(55.6)$ \\
\hline \multirow[t]{2}{*}{ Total $(\mathrm{N}=20)$} & $S$ & $1(5)$ & $13(65)$ & $15(75)$ & $6(30)$ & $10(50)$ & 17(85) & $14(70)$ & $9(45)$ & $17(85)$ & $15(75)$ & $6(30)$ \\
\hline & I & 1(5) & $3(15)$ & $1(5)$ & $4(20)$ & & & $3(15)$ & $3(15)$ & $1(5)$ & $1(5)$ & $3(15)$ \\
\hline
\end{tabular}

Among the total isolates $(n=69)$ multi drug resistance $(M D R)$ was observed in $62(89.9 \%)$ of bacteria isolated. In Gram-negative bacteria MDR were observed in $45 / 49$ (91.8\%) while gram-positive bacteria were observed in $17 / 20$ (85\%) respectively (Table6).

Table6. Multi drug resistance pattern of bacterial isolates from pregnant women with UTI attended antenatal care at HGH, Hargeisa, Somaliland, 2020 ( $\mathrm{n}=69$ )

\begin{tabular}{|c|c|c|c|c|c|c|c|c|}
\hline \multirow[t]{2}{*}{ Isolates } & \multicolumn{3}{|c|}{ Frequency (\%) } & \multirow[b]{2}{*}{ R4 } & \multirow[b]{2}{*}{ R5 } & \multirow[b]{2}{*}{ R6 } & \multirow[b]{2}{*}{$\geq \mathrm{R7}$} & \multirow[b]{2}{*}{ MDR } \\
\hline & Total & R2 & R3 & & & & & \\
\hline Gram-negative & $49(71)$ & $1(2)$ & $9(18.4)$ & 10(20.4) & 17(34.7) & $4(8.2)$ & $4(8.2)$ & $45(91.8)$ \\
\hline E. coli & $30(61.2)$ & $1(3.3)$ & $7(23.3)$ & $5(16.7)$ & $11(40)$ & $3(6.7)$ & $1(3.3)$ & $28(57.1)$ \\
\hline K. pneumonia & $6(12.2)$ & & 1(16.7) & & 2(33.3) & & 2(33.3) & $5(10.2)$ \\
\hline P. aeruginosa & $5(10.2)$ & & & $3(60)$ & $2(40)$ & & & $5(10.2)$ \\
\hline P. mirabilis & $4(8.2)$ & & $1(25)$ & $0(0)$ & $1(25)$ & & $1(25)$ & $3(6.1)$ \\
\hline Citrobacter spp. & $3(6.1)$ & & & $2(66.7)$ & 1(33.3) & & & $3(6.1)$ \\
\hline M. morganii & $1(2)$ & & & & & $1(100)$ & & $1(2)$ \\
\hline Gram-positive & 20(29) & $2(10)$ & $1(5)$ & $8(40)$ & $2(10)$ & $3(15)$ & $1(5)$ & $17(85)$ \\
\hline CoNS & $11(55)$ & & & $7(63.6)$ & $1(9.1)$ & $1(9.1)$ & & $9(45)$ \\
\hline S. aureus & $9(45)$ & $2(22.2)$ & $1(11.1)$ & $1(11.1)$ & $1(11.1)$ & $2(22.2)$ & $1(11.1)$ & $8(40)$ \\
\hline Total & $69(100)$ & $3(4.4)$ & $10(14.5)$ & $18(26.1)$ & $19(27.5)$ & $9(13)$ & $3(4.4)$ & $62(89.9)$ \\
\hline
\end{tabular}

$\mathrm{R} 2=$ resistance to two drugs, $\mathrm{R} 3$ = resistance to three drugs, R4= resistance to four drugs, R5=resistance to five drugs, R6=resistance to six drugs and $\geq \mathrm{R} 7=$ resistance to seven and more drugs, MDR=multiple drug resistance.

In bivariate analysis, Age of respondents [COR=2.600 95\% Cl: 0.725-9.319], Educational status [COR=2.780 95\% Cl: 0.965-8.006], Family income [COR=3.559 95\% Cl: 0.978-12.954], History of catheterization [COR=3.154, 95\% Cl: 1.388-7.170], History of abortion [COR=2.220, 95\% Cl: 0.756-6.517] and History of previous UTI [COR=3.31, 95\% Cl: $1.740-6.300$ ] were found to be significantly associated with UTI among pregnant women and were to be a candidate for multivariate logistic regression analysis.

The result of multivariate analysis revealed that pregnant women with no formal education were 3.2 more likely to have UTI than those with higher education (> grade 12) [ AOR=3.183 95\% Cl: 1.027-9.866],

family income $\leq \$ 100$ was 5.2 times higher risk of having UTI than those with family income $>\$ 300$ [AOR=5.225 95\% Cl: $1.270-21.500]$, the odds of having UTI among pregnant women who have previously indwelling catheter were 3.2 times higher than the odds in pregnant women who have not previously indwelling catheter [AOR=3.216,95\% Cl: 1.287-8.038], pregnant women who have previous history of UTI were 3.7 more likely to occur the UTI compared with pregnant women that have not the previous history of UTI [AOR=3.734, 95\% Cl: 1.855-7.515](Table7). 
Table7: Bivariate and multivariate analysis for the assessment of factors associated with UTI among pregnant women attending antenatal care at HGH, Hargeisa, Somaliland, $2020(n=422)$. 


\begin{tabular}{|c|c|c|c|c|c|c|c|}
\hline \multirow[t]{2}{*}{ Variables } & & \multicolumn{2}{|l|}{ UTI } & \multirow[t]{2}{*}{$\operatorname{COR}(95 \% \mathrm{Cl})$} & \multirow{2}{*}{$\begin{array}{l}P \\
\text { values }\end{array}$} & \multirow[t]{2}{*}{$\operatorname{AOR}(95 \% \mathrm{Cl})$} & \multirow{2}{*}{$\begin{array}{l}P \\
\text { values }\end{array}$} \\
\hline & & Yes (\%) & No (\%) & & & & \\
\hline \multirow[t]{6}{*}{ Age (in years) } & $\leq 19$ & $7(41.2)$ & $10(58.8)$ & $\begin{array}{l}2.600(0.725- \\
9.319)\end{array}$ & 0.142 & $\begin{array}{l}2.446(0.609- \\
9.818)\end{array}$ & 0.207 \\
\hline & $20-25$ & 17(19.3) & $71(80.7)$ & $\begin{array}{l}0.889(0.331- \\
2.389)\end{array}$ & 0.816 & $\begin{array}{l}0.903(0.313- \\
2.608)\end{array}$ & 0.851 \\
\hline & $26-30$ & $20(16.8)$ & $99(83.2)$ & $\begin{array}{l}0.750(0.286- \\
1.966)\end{array}$ & 0.559 & $\begin{array}{l}0.813(0.285- \\
2.316)\end{array}$ & 0.698 \\
\hline & $31-35$ & $18(14.4)$ & 107(85.6) & $\begin{array}{l}0.625(0.236- \\
1.652)\end{array}$ & 0.343 & $\begin{array}{l}0.606(0.212- \\
1.732)\end{array}$ & 0.350 \\
\hline & $36-40$ & $0(0)$ & $40(100)$ & $0.000(0.000)$ & 0.998 & $0.000(0.000)$ & 0.997 \\
\hline & $41-45$ & $7(21.2)$ & $26(78.8)$ & I & & & \\
\hline \multirow[t]{2}{*}{ Residence } & Urban & $66(16.6)$ & $332(83.4)$ & $\begin{array}{l}1.392[0.403- \\
4.800]\end{array}$ & 0.601 & & \\
\hline & Rural & $3(12.5)$ & $21(87.5)$ & I & & & \\
\hline \multirow[t]{4}{*}{ Marital status } & Widowed & $2(25)$ & $6(75)$ & I & & & \\
\hline & Married & $66(16.2)$ & $341(83.8)$ & $\begin{array}{l}0.581[0.115- \\
2.940]\end{array}$ & 0.511 & & \\
\hline & Divorced & $0(0)$ & $2(100)$ & $0.000[0.000]$ & 0.999 & & \\
\hline & Separated & $1(20)$ & $4(80)$ & $\begin{array}{l}0.750[0.050- \\
11.311]\end{array}$ & 0.835 & & \\
\hline \multirow[t]{4}{*}{ Educational status } & No formal education & 20 & 59 & $\begin{array}{l}2.780[0.965- \\
8.006]\end{array}$ & 0.058 & $\begin{array}{l}3.183[1.027- \\
9.866]\end{array}$ & 0.045 \\
\hline & Primary education (1-8) & 29 & 156 & $\begin{array}{l}1.524[0.555- \\
4.183\end{array}$ & 0.413 & $\begin{array}{l}1.624[0.553- \\
4.766]\end{array}$ & 0.378 \\
\hline & $\begin{array}{l}\text { Secondary education (9- } \\
\text { 12) }\end{array}$ & 15 & 97 & $\begin{array}{l}1.268[0.432- \\
3.719]\end{array}$ & 0.665 & $\begin{array}{l}1.767[0.552- \\
5.658]\end{array}$ & 0.338 \\
\hline & Higher Education (>12) & 5 & 41 & I & & & \\
\hline \multirow[t]{4}{*}{ Family Income } & $\leq \$ 100$ & 18 & 59 & $\begin{array}{l}3.559[0.978- \\
12.954]\end{array}$ & 0.054 & $\begin{array}{l}5.225[1.270- \\
21.500]\end{array}$ & 0.022 \\
\hline & $\$ 101-200$ & 32 & 159 & $\begin{array}{l}2.348[0.680- \\
8.103]\end{array}$ & 0.177 & $\begin{array}{l}2.940[0.761- \\
11.352]\end{array}$ & 0.118 \\
\hline & $\$ 201-300$ & 16 & 100 & $\begin{array}{l}1.867[0.513- \\
6.793]\end{array}$ & 0.344 & $\begin{array}{l}2.839[0.694- \\
11.615]\end{array}$ & 0.147 \\
\hline & $>\$ 300$ & 3 & 35 & I & & & \\
\hline \multirow[t]{4}{*}{ Occupational status } & House wife & 47 & 196 & $\begin{array}{l}0.839[0.169- \\
4.171]\end{array}$ & 0.830 & & \\
\hline & Merchant & 12 & 80 & $\begin{array}{l}0.525[0.097- \\
2.830]\end{array}$ & 0.453 & & \\
\hline & Governmental employee & 8 & 70 & $\begin{array}{l}0.400[0.071- \\
2.264]\end{array}$ & 0.300 & & \\
\hline & Student & 2 & 7 & 1 & & & \\
\hline \multirow[t]{3}{*}{ Gestational Period } & $1^{\text {st }}$ trimester & 16 & 71 & I & & & \\
\hline & $2^{\text {nd }}$ trimester & 23 & 114 & $\begin{array}{l}0.895[0.443- \\
1.809]\end{array}$ & 0.758 & & \\
\hline & $3^{\text {rd }}$ trimester & 30 & 168 & $\begin{array}{l}0.792[0.407- \\
1.544]\end{array}$ & 0.494 & & \\
\hline \multirow[t]{2}{*}{ Gravida } & Primigravida & 12 & 44 & I & & & \\
\hline & Multigravida & 57 & 309 & $\begin{array}{l}0.676[0.337- \\
1.359]\end{array}$ & 0.272 & & \\
\hline \multirow[t]{2}{*}{ History of Catheterization } & No & 59 & 335 & I & & & \\
\hline & Yes & 10 & 18 & $\begin{array}{l}3.154[1.388- \\
7.170]\end{array}$ & 0.006 & $\begin{array}{l}3.216[1.287- \\
8.038]\end{array}$ & 0.012 \\
\hline History of diabetes mellitus & No & 67 & 345 & I & & & \\
\hline
\end{tabular}




\begin{tabular}{|c|c|c|c|c|c|c|c|}
\hline & Yes & 2 & 8 & $\begin{array}{l}1.287[0.267- \\
6.196]^{0}\end{array}$ & 0.753 & & \\
\hline \multirow[t]{2}{*}{ History of abortion } & No & 64 & 341 & 1 & & & \\
\hline & Yes & 5 & 12 & $\begin{array}{l}2.220[0.756- \\
6.517]^{0}\end{array}$ & 0.147 & $\begin{array}{l}2.183[0.672- \\
7.092]^{[0}\end{array}$ & 0.194 \\
\hline \multirow{2}{*}{$\begin{array}{l}\text { History of obstetric and gynecologic } \\
\text { surgery }\end{array}$} & No & 67 & 346 & 1 & & & \\
\hline & Yes & 2 & 7 & $\begin{array}{l}1.475[0.300- \\
7.258]\end{array}$ & 0.632 & & \\
\hline \multirow[t]{2}{*}{ History of premature labor } & No & 68 & 348 & I & & & \\
\hline & Yes & 1 & 5 & $\begin{array}{l}1.024[0.118- \\
8.899]^{0}\end{array}$ & 0.983 & & \\
\hline \multirow[t]{2}{*}{ History of previous UTI } & No & 51 & 319 & 1 & & & \\
\hline & Yes & 18 & 34 & $\begin{array}{l}3.311[1.740- \\
6.300]\end{array}$ & 0.000 & $\begin{array}{l}3.734[1.855- \\
7.515]\end{array}$ & 0.000 \\
\hline
\end{tabular}

$\mathrm{I}=$ reference, $\mathrm{AOD}=$ adjusted odds ratio, $\mathrm{COR}=$ crude odds ratio, $\mathrm{N}=$ number, $\mathrm{UTI}=$ Urinary tract infection

\section{Discussion}

The overall prevalence of UTI in pregnant women in this study was $16.4 \%$. This is comparable to the prevalence of UTI reported in in Mwanza City, Tanzania $16.8 \%^{10}$, in Nairobi, Kenya $15.7 \%^{11}$, in Kano, Northern Nigeria $15.8 \%{ }^{12}$ and in Bangalore, India $15 \%{ }^{13}$.

Whereas a higher prevalence was reported in Ambo Central Ethiopia $18.7 \%{ }^{14}$, in Derna City Libya $49.3 \%{ }^{15}$, in Ismailia, Egypt $29 \%{ }^{16}$, in Benin city, Nigeria $21 \% 17$, in Saudi Arabia $53.5 \%{ }^{18}$, in Nepal $37.8 \%{ }^{19}$ respectively. A lower prevalence was reported from Iran $13.1 \%{ }^{20}$, Gondar Northwest Ethiopia $10.4 \% 21$, Korela India $13.4 \%^{22}$, Khartoum Sudan $14 \%{ }^{23}$. This variation in prevalence might be due to across different studies from one country to another and among regions of the same country might be attributed to the difference in associated factors, sample size, social habits of the community, the standard of personal hygiene and education.

The prevalence of UTI among symptomatic and asymptomatic pregnant women in this study was $9.5 \%$ and $6.9 \%$ respectively. The occurrence of UTI case among asymptomatic was in line with the previous study done in Cameroon $7.8 \%{ }^{24}$, in Kanpur, India $7.3 \%{ }^{25}$ and in Makkah, Saudi Arabia $8 \%{ }^{26}$, On the other hand, a low prevalence of $0.13 \%$ In meta-analysis in Iran ${ }^{27}$, in Colombo, Sri Lanka $3.6 \%{ }^{28}$ and in Ghana $5.5 \%{ }^{29}$ was reported asymptomatic UTI. While higher prevalence was recorded in Hawassa, Southern $21.2 \%{ }^{30}$, in Bangladesh $10.2 \%{ }^{31}$ and in Nairobi, Kenya $21.5 \%{ }^{32}$.

In this study the symptomatic study was $9.5 \%$, These result of symptomatic UTI were agree with a study conducted from in Khartoum Sudan $12.1 \%{ }^{23}$, in Makkah, Saudi Arabia $12 \%^{26}$ and in Northeastern Ethiopia 11.9\% ${ }^{33}$. But higher prevalence rate in Mekelle Northern Ethiopia $21.1 \%{ }^{34}$, in Bangladesh $17.9 \%{ }^{35}$, in in Goba and Sinana Woredas, Bale Zone, Southeast Ethiopia $35.3 \%{ }^{36}$ and in South-western Uganda $35 \%{ }^{37}$. The differences may be the variation of methodologies and study populations might affect prevalence in different sites.

In this study, Gram-negative bacteria isolates were more prevalent (71\%) than Gram-positive bacteria isolates (29\%). A similar finding was found from Ambo town, Central Ethiopia $69.6 \%$ and $30.4 \%$ for Gram negative and Gram positive pathogens ${ }^{14}$, in Tanzania also Gram negative bacteria and gram positive bacteria were reported $61.9 \%$ and $38.1 \%{ }^{38}$ respectively. This could be due to the presence of unique structure in Gram negative bacteria which help for attachment to the uroepithelial cells and prevent bacteria from urinary lavage, allowing for multiplication and tissue invasion-resulting in invasive infection and pyelonephritis in pregnancy ${ }^{39}$.

Among isolated pathogens, were E. coli was the most predominant bacteria $43.5 \%$, which is similar with previous studies in Ambo town, Central Ethiopia $46.4 \%$ of isolated cases ${ }^{14}$, in Bangalore, India $43.9 \%{ }^{40}$ and in Nairobi, Kenya $40.0 \%$ was reported ${ }^{41}$. However, it was lower than reported in the previous studies conducted in different countries, which was India $53.8 \%$, Italy $57.1 \%$ and Iran $57.25 \% 22,42,43$. E. coli is the most common microorganism in the vaginal and rectal area. Because of anatomical and functional changes and difficulty of maintaining personal hygiene during pregnancy, may increase the risk of acquiring UTI from E. coli ${ }^{44}$. The second most common isolate was CONS $16 \%$ and comparable findings have been reported in different studies like in Karamara Hospital Jigjiga, Eastern Ethiopia $12 \%{ }^{45}$, Saint Paul's Hospital Millennium Medical College, Addis Ababa, Ethiopia $14.3 \%$ and in Dil Chora Referral Hospital, Dire Dawa, Eastern Ethiopia $19.2 \%{ }^{47}$.

In this study, susceptibility pattern of Gram-negative bacteria showed that most of the isolates were sensitive to meropenem (95.9\%), ceftriaxone (79.6\%), norfloxacin (77.5\%), gentamicin (75.5\%), nitrofurantoin (75.5\%) and ciprofloxacin (71.4\%) and comparable studies conducted in different study area like in Addis Ababa, Ethiopia that showed highly sensitive to meropenem (75.2\%), nitrofurantoin (93.1\%), gentamicin (85.2\%), ceftriaxone (82.2\%), cefuroxime (79.3\%), and ciprofloxacin (75.2\%) ${ }^{46}$ and in South Nigeria showed sensitive to gentamicin (53\% - $\left.100 \%\right)$, imipenam (67\%-93\%), ciprofloxacin (between $57 \%-75 \%)^{48}$, In Hawassa, Southern Ethiopia the present study, $80 \%$ of Gram negative bacteria were susceptible to meropenem, ciprofloxacin, gentamicin, nitrofurantoin, and norfoxacin ${ }^{49}$. In this study, the highest resistance was shown to ampicillin (93\%-100\%) among gram-negative bacteria, this due to the drug 
is with low cost and often can be purchased without prescription in different areas. This implies that ampicillin cannot be used as empirical therapy for urinary tract infection particularly in the study area. This also agrees with the study done in Karamara Hospital Jigjiga, Eastern Ethiopia 45 .

In this study the other drugs also showed highly resistance to Gram-negative were tetracycline (71.4\%), trimethoprim-sulfamethoxazole (57.1\%), amoxicillin clavulanic acid (55.1\%) and nalidixic acid (51\%) and this agree the other study done in Dil Chora Referral Hospital, Dire Dawa, Eastern Ethiopia AMP (89.5\%), amoxicillin (73.7\%), and TTC (73.7\%), NA (52.6\%), except nitrofurantoin (57.9\%) ${ }^{47}$ and in Mbarara Regional Referral Hospital, South-western Uganda were highly resistant to Amoxicillin, Ampicillin, and Amoxicillin/Clavulanic acid at $95.7 \%, 95.0 \%$, and $72.9 \%{ }^{37}$. The findings of this study is not in line with the reports from Kenya ${ }^{41}$. These differences could be due to variations in antibiotic prescription patterns across various countries.

In this current study, the Gram-negatives, the predominant isolate was E. coli, which is resistant to ampicillin (93.3\%), TTC (73.3\%), SXT (60\%), nalidixic acid (53.3\%) and AMC (46.7\%). Similar findings have been reported from previous studies in Dire Dawa, Eastern Ethiopia, South-western Uganda and Addis Ababa, Ethiopia $37,46,47$. The other isolated Gram negative bacteria include $K$. pneumonia showed more than $65 \%$ sensitive to meropenem, gentamicin, ceftriaxone, ciprofloxacin and nitrofurantoin this agreed with the study done in South-western Uganda ${ }^{37}$, K. pneumoniae was $100 \%$ resistant to ampicillin, Similar findings were done in Adigrat General Hospital, Northern Ethiopia and Karamara Hospital Jigjiga, Eastern Ethiopia ${ }^{45,50}$.

In this study, the Gram positive bacterial isolates were relatively sensitive to erythromycin, cefoxitin, ceftriaxone, nitrofurantoin, gentamicin, ciprofloxacin and each accounted $85 \%, 85 \%, 75 \%, 75 \%, 70 \%$ and $65 \%$. This was comparable with the finding from Ivory Coast, Dire Dawa, Eastern Ethiopia and Gonder Ethiopia 47,51,52 However, in contrast with study report from Southern Ethiopia, which ceftriaxone was $100 \%$ resistant to gram-positive bacteria 53 .

In this study, Gram positive bacteria showed highly resistance to ampicillin $90 \%$ and tetracycline $55 \%$. This could be due to the infrequent use of the drug in the study area. Comparable result was reported in Gonder Ethiopia ${ }^{52}$, in Lagos, Nigeria, and Benishangul Gumuz Region, Western Ethiopia ${ }^{54}$. Coagulase negative staphylococci, which were the predominant isolates from Gram-positives $55 \%$ and was found $63 \%$ to $81 \%$ sensitive to erythromycin, cefoxitin, ceftriaxone, nitrofurantoin gentamicin and ciprofloxacin. However, in contrast was shown nitrofurantoin to $26.7 \%$ resistance in study done in Ethiopia 55 , while comparable studies done in Hawassa, Ethiopia ${ }^{49}$.

In this study, S. aureus which constituted for $45 \%$ of the gram positive bacteria showed $66.7 \%-88.9 \%$ were sensitive to erythromycin, cefoxitin, ceftriaxone, nitrofurantoin, ciprofloxacin and gentamicin, this agree with study done in Hawassa, Ethiopia, Benishangul Gumuz Region, Western Ethiopia and Nairobi, Kenya ${ }^{41,49,54}$. In contrast to research done in Addis Ababa, Ethiopia, which erythromycin was highly resistant $60 \%{ }^{46}$. However, this study showed $100 \%$ ampicillin to S. aureus, similar study done in Jigjiga, Ethiopia, Dire Dawa, Eastern Ethiopia and Addis Ababa, Ethiopia $45,47,56$. This is caused by use of empirical treatment against bacterial infections of the urinary tract infection in the study area. This implies that ampicillin cannot be used as empirical therapy for urinary tract infection particularly in the study area.

In this study, MDR was seen in $89.9 \%$ of all bacteria isolated. Our finding is higher than studies done in same regions of Ethiopia like $57.1 \%$ in Addis Ababa, and $73 \%$ in Mekelle ${ }^{34,46}$, in Tanzania $77 \%{ }^{38}$ and in Eastern Uganda $77.5^{57}$. . Our finding is lower than studies done in South-South Nigeria $100 \%$, in Kenya $96 \%{ }^{11}$ and same regions in Ethiopia like in Dire Dawa 100\%, Gondar 95\% and Jigjiga $96 \%$ 21,45,47. This indicates that multi drug resistance was found to be very high to the commonly used antibiotics. Antibiotic resistance has been recognized as the consequence of antibiotic use and abuse ${ }^{58}$. Therefore, the reasons for this alarming phenomenon might be inappropriate and incorrect administration of antimicrobial agents in empiric therapies and lack of appropriate infection control strategies, which can cause a shift to increase prevalence of resistant organisms in the community.

In the present study, the result of multivariable logistic regression models revealed that socio-demographic factors among pregnant women were statistically significance with no formal education and low level of family income $(\leq \$ 100)[P=0.045, A O R=3.183(1.027,9.866)]$ and $[P=0.022, A O R=5.225(1.270$, 21.500)]. The non-formal education was agreed with study done in Goba and Sinana Woredas, Bale Zone, Southeast Ethiopia [AOR $=6.617 ; \mathrm{Cl}=1.87-9.94]$

${ }^{36}$. in contract with low level education the studies done in Medan, Indonesia, and Uyo, Nigeria ${ }^{48,59}$. Low-income status was another factor that was related with high prevalence of UTI among pregnant women. A similar finding was reported in other studies on pregnant women in Dire Dawa, Eastern Ethiopia and in Adigrat General Hospital, Northern Ethiopia 47,50 . This could be due to the relation of low socioeconomic status with nutrition and immunity especially in pregnant women. In contrast studies were done in Jigjiga, Ethiopia, Medan, Indonesia, and Northeastern Ethiopia ${ }^{33,45,59}$

In the study, obstetrics and clinical characteristics were shown that, highly significant proportion of UTI was recorded among those study subjects with prior history of UTI. The multivariate logistic regression analysis of current study showed that 3.7 more likely to occur the UTI compared with pregnant women that have not the previous history of UTI $[P=0.000$ AOR=3.734 $(1.855,7.515)]$. This finding is similar with report from Uganda $(P=0.002), L i b y a(P=0.00)$, Egypt $(P=0.001)$, India $(P=0.0423)$, and same regions of Ethiopia like Gondar, ( $p$-value=0.001), Dire Dewa ( $p$-value=0.006) and Addis Ababa $(P=$ $0.004)^{15,21,22,37,46,47,60}$. The possible explanation for this association could be due to the existence of antibiotic-resistant strains from the previous infection.

In the current study, participants with the previous history of indwelling catheterization had about 3.2 times chance of developing UTI [P= $0.012 \mathrm{AOR}=3.216$ $(1.287,8.038)]$ among pregnant women. This finding agrees with similar reports from Northeastern Ethiopia, Addis Ababa, Ethiopia and Gonder Ethiopia $21,33,46$. This could be due to long duration of catheterization, frequent catheterization or contamination during inserting catheters. However other studies done in Dire Dawa, Eastern Ethiopia and Jigjiga Eastern Ethiopia disagreed of this study ${ }^{45,47 .}$

In the present study, there was no statistical significant association between prevalence of UTI among pregnant women and maternal age, residence, marital status, occupation, gestational period, gravidity, History of diabetes mellitus, History of abortion History of obstetric and gynecologic surgery and History of 
premature labor. this results were agreed the report from Bangladesh ${ }^{35}$, Nairobi, Kenya except the maternal age ${ }^{32}$, Nigeria ${ }^{48}$, Goba and Sinana Ethiopia ${ }^{36}$, Dire Dawa, Eastern Ethiopia ${ }^{47}$ and Addis Ababa, Ethiopia except history of abortion ${ }^{46}$.

\section{Conclusion}

The overall prevalence was $16.4 \%$, eight species of bacteria were isolated both gram negative and gram positive. Majority of the isolates were resistant to the commonly prescribed antibiotics, therefore culture and antibiotic susceptibility testing was recommended before giving treatment to prevent antimicrobial resistance and health information dissemination to the patients recommended to avoid self-medication practice

\section{Abbreviations}

ANC Antenatal care

HGH Hargeisa Group Hospital

MDR Multi drug resistance

UTI Urinary tract infection

\section{Declarations}

\section{Data Availability}

The datasets used and analyzed in the current study are available from the corresponding author on reasonable request.

\section{Ethics approval and consent to participate}

Ethical approval was obtained from the institutional review board of Hawassa University, College of medicine and health sciences (Ref No: IRB/231/11). Informed written consent was obtained from all participants. All methods were carried out in accordance with relevant guidelines and regulations. Clinicians were communicated the findings of culture and sensitivity tests.

\section{Consent for publication}

Individual data such as images and videos did not accompany this particular manuscript and hence consent for publication is not applicable.

\section{Availability of data and materials}

The datasets used and analyzed during the current study are available from the corresponding author on reasonable request.

\section{Competing interests}

The authors declare that they have no competing interests for this work.

\section{Funding}

Not applicable.

\section{Acknowledgments}

We are grateful to Hawassa University Comprehensive Specialized Hospital microbiology laboratory staffs and study participants for their contribution to this research work.

\section{Authors' contributions}

M.D.O., D.Y.R. and A.H.A conceived the study and participated in data analysis. A.H.A. carried out the lab work, M.D.O. wrote the manuscript. All authors read and approved the final manuscript.

\section{Additional information}

Competing Interests: The authors declare no competing interests.

Publisher's note: Springer Nature remains neutral with regard to jurisdictional claims in published maps and institutional affiliations.

\section{References}

1. Amarasinghe, N. et al. Bacterial profile and antibiotic susceptibility pattern of adult lower respiratory tract infections in Colombo, Sri Lanka. Journal of Health and Social Sciences, 3, 27-36 (2018). 
2. Bigna, T. S. et al. Prevalence of respiratory bacterial infections in people with lower respiratory tract infections in Africa: the BARIAFRICA systematic review and meta-analysis protocol. BMJ open, 8, e023592 (2018).

3. Tchatchouang, S. et al. Bacterial Aetiologies of Lower Respiratory Tract Infections among Adults in Yaoundé, Cameroon.BioMed research internationa/2019(2019).

4. Cheesbrough, M. District laboratory practice in tropical countries. (Cambridge university press, United States of America, New York, 2006).

5. Tadesse, S. et al. Prevalence, antimicrobial susceptibility profile and predictors of asymptomatic bacteriuria among pregnant women in Adigrat General Hospital, Northern Ethiopia. BMC research notes, 11, 740 (2018).

6. Gessese, Y. A. et al. Urinary pathogenic bacterial profile, antibiogram of isolates and associated risk factors among pregnant women in Ambo town, Central Ethiopia: a cross-sectional study. Antimicrobial resistance and infection control, 6, 132 (2017).

7. Bauer, A. W., Kirby, W. M. M., Sherris, J. C. \& Turck, M. Antibiotic Susceptibility Testing by a Standardized Single Disk Method. American Journal of Clinical Pathology, 45, 493-496 (1966).

8. CLSI. Performance standards for antimicrobial susceptibility testing. 30th ed. (Wayne,PA, 2020).

9. Bursac, Z., Gauss, C. H., Williams, D. K. \& Hosmer, D. W. Purposeful selection of variables in logistic regression. Source Code Biol Med, 3, 17-17 (2008).

10. Kaduma, J. et al. Urinary Tract Infections and Preeclampsia among Pregnant Women Attending Two Hospitals in Mwanza City, Tanzania: A 1:2 Matched Case-Control Study. BioMed research international 2019, 3937812 (2019).

11. Onyango, H. A., Ngugi, C., Maina, J. \& Kiiru, J. Urinary Tract Infection among Pregnant Women at Pumwani Maternity Hospital, Nairobi, Kenya: Bacterial Etiologic Agents, Antimicrobial Susceptibility Profiles and Associated Risk Factors. Advances in Microbiology, 8 (03), 175 (2018).

12. Ali, M. \& Abdallah, M. S. Prevalence of Urinary Tract Infection among Pregnant Women in Kano, Northern Nigeria. Arch Reprod Med Sex Health, 2 (1), $23-$ 29 (2019).

13. Rudri Bai, I., Deepthi, M. \& Dharmavijaya, M. Prevalence of urinary tract infection in antenatal period at tertiary care hospital in rural Bangalore. International Journal of Clinical Obstetrics and Gynaecology, 2 (4), 69-71 (2018).

14. Gessese, Y. A. et al. Urinary pathogenic bacterial profile, antibiogram of isolates and associated risk factors among pregnant women in Ambo town, Central Ethiopia: a cross-sectional study. Antimicrobial Resistance \& Infection Control, 6 (1), 1-10 (2017).

15. Younis, M., Ajroud, S., Elgade, L. H., Uahua, A. S. \& Elzahaf, R. A. Prevalence of Urinary Tract Infection among Pregnant Women and Its Risk Factor in Derna City. Scholars International Journal of Obstetrics and Gynecology a2 (8), 219-223(2019).

16. Mohamed, N. R., Omar, H. \& Abdallah, I. Prevalence and Risk Factors of Urinary Tract Infection among Pregnant Women in Ismailia City, Egypt. IOSR Journal of Nursing and health Science, 06 (03), 62-72 (2017).

17. Mordi, R. M., Burke, M. E., Odjadjare, E. E., Enabulele, S. A. \& Umeh, O. J. Prevalence of urinary tract infections (UTI) among pregnant women in university of Benin teaching hospital (UBTH) Benin City, Nigeria. J Asian Sci Res, 5 (4), 198-204 (2015).

18. El-Kashif, M. M. L. Urinary Tract Infection among Pregnant Women and its Associated Risk Factors: A Cross-Sectional Study. Biomedical and Pharmacology Journal, 12 (4), 2003-2010 (2019).

19. Thakur, S. \& Nagpal, K. L. Urinary Tract Infection in Pregnant Women at Kathmandu, Nepal. Journal of Medical Care Research and Review, 3 (9), $454-458$ (2020).

20. Rejali, M. \& Ahmadi, S. S. Prevalence and Risk Factors of Urinary Tract Infection among Pregnant Women in Shahrekord, Iran. International Journal of Epidemiologic Research, 6 (2), 55-59 (2019).

21. Alemu, A. et al. Bacterial profile and drug susceptibility pattern of urinary tract infection in pregnant women at University of Gondar Teaching Hospital, Northwest Ethiopia. BMC research notes, 5 (1), 1-7 (2012).

22. Srinath, M., Shajina, M. \& Fasalu Rahiman, O. Etiology and prevalence of urinary tract infections among pregnant women in Kerala. International Journal of Community Medicine and Public Health, 5 (8), 3298 (2018).

23. Hamdan, H. Z., Ziad, A. H. M., Ali, S. K. \& Adam, I. Epidemiology of urinary tract infections and antibiotics sensitivity among pregnant women at Khartoum North Hospital. Annals of clinical microbiology and antimicrobials, 10 (1), 1-5 (2011).

24. Mokube, M. N., Atashili, J., Halle-Ekane, G. E., Ikomey, G. M. \& Ndumbe, P. M. Bacteriuria amongst pregnant women in the Buea Health District, Cameroon: Prevalence, predictors, antibiotic susceptibility patterns and diagnosis. PLoS One, 8 (8), e71086 (2013).

25. Sujatha, R. \& Nawani, M. Prevalence of asymptomatic bacteriuria and its antibacterial susceptibility pattern among pregnant women attending the antenatal clinic at Kanpur, India. Journal of clinical and diagnostic research: JCDR, 8 (4), DC01-DC03 (2014).

26. Faidah, H. S., Ashshi, A. M., El-Ella, G. A. A., Al-Ghamdi, A. K. \& Mohamed, A. M. Urinary tract infections among pregnant women in Makkah, Saudi Arabia. Biomedical And Pharmacology Journal, 6, 01-07 (2015).

27. Ghafari, M., Baigi, V., Cheraghi, Z. \& Doosti-Irani, A. The prevalence of asymptomatic bacteriuria in Iranian pregnant women: a systematic review and meta-analysis. PloS one, 11 (6), e0158031 (2016).

28. Perera, J., Randeniya, C., Perera, P., Gamhewage, N. \& Jayalatharchchi, R. Asymptomatic bacteriuria in pregnancy: prevalence, risk factors and causative organisms. Sri Lankan Journal of Infectious Diseases, 2 (1), 42-46 (2012).

29. Labi, A. K., Yawson, A. E., Ganyaglo, G. Y. \& Newman, M. J. Prevalence and Associated Risk Factors of Asymptomatic Bacteriuria in Ante-Natal Clients in a Large Teaching Hospital in Ghana. Ghana Med J, 49 (3), 154-158 (2015).

30. Tadesse, E., Teshome, M., Merid, Y., Kibret, B. \& Shimelis, T. Asymptomatic urinary tract infection among pregnant women attending the antenatal clinic of Hawassa Referral Hospital, Southern Ethiopia. BMC research notes, 7 (1), 155 (2014). 
31. Jubaida, N. et al. Prevalence of asymptomatic bacteriuria in pregnant women. Journal of Armed Forces Medical College, Bangladesh, 9 (2), $64-69$ (2013).

32. Ayoyi, A. O., Kikuvi, G., Bii, C. \& Kariuki, S. Prevalence, aetiology and antibiotic sensitivity profile of asymptomatic bacteriuria isolates from pregnant women in selected antenatal clinic from Nairobi, Kenya. Pan Afr Med J, 26 (1), 41-41 (2017).

33. Belete, M. A. Bacterial profile and ESBL screening of urinary tract infection among asymptomatic and symptomatic pregnant women attending antenatal care of northeastern Ethiopia region. Infection and Drug Resistance, 13, 2579 (2020).

34. Gebremariam, G. et al. Bacteriological profile, risk factors and antimicrobial susceptibility patterns of symptomatic urinary tract infection among students of Mekelle University, northern Ethiopia. BMC Infect Dis, 19 (1), 950-950 (2019).

35. Uddin, M. N. \& Khan, T. Prevalence of urinary tract infection among pregnant women at Ibrahim Iqbal Memorial Hospital, Chandanaish, Bangladesh. Am J Clin Med Res, 4 (3), 47-51 (2016).

36. Taye, S., Getachew, M., Desalegn, Z., Biratu, A. \& Mubashir, K. Bacterial profile, antibiotic susceptibility pattern and associated factors among pregnant women with Urinary Tract Infection in Goba and Sinana Woredas, Bale Zone, Southeast Ethiopia. BMC research notes, 11, 799 (2018).

37. Bahati, J. et al. Prevalence and Bacteriology of Symptomatic Urinary Tract Infection among Pregnant women at Mbarara Regional Referral Hospital, South-western Uganda.1-13(2020)

38. Moyo, S. J., Aboud, S., Kasubi, M., Lyamuya, E. F. \& Maselle, S. Y. Antimicrobial resistance among producers and non-producers of extended spectrum beta-lactamases in urinary isolates at a tertiary Hospital in Tanzania. BMC Research Notes, 3 (1), 1-5 (2010).

39. Nasiriamiri, F., Rooshan, M., Ahmady, H. \& Soliamani, M. Hygiene practices and sexual activity associated with urinary tract infection in pregnant women. Eastern Mediterranean health journal = La revue de santé de la Méditerranée orientale = al-Majallah al-ṣiḥhịyah li-sharq al-mutawassit 15, 104-110(2008).

40. Sibi, G., Kumari, P. \& Kabungulundabungi, N. Antibiotic sensitivity pattern from pregnant women with urinary tract infection in Bangalore, India. Asian Pacific Journal of Tropical Medicine, 7, S116-S120 (2014).

41. Nabbugodi, W. F., Gichuhi, J. W. \& Mugo, N. Prevalence of Urinary TractInfection, Microbial Aetiology, and Antibiotic Sensitivity Pattern among Antenatal Women Presenting with Lower Abdominal Pains at Kenyatta National Hospital, Nairobi, Kenya. The Open Access Journal of Science and Technology, 3 (3), 1-6 (2015).

42. De Francesco, M. A., Ravizzola, G., Peroni, L., Negrini, R. \& Manca, N. Urinary tract infections in Brescia, Italy: etiology of uropathogens and antimicrobial resistance of common uropathogens. Medical science monitor, 13 (6), BR136-BR144 (2007).

43. Amiri, M. et al. Prevalence of urinary tract infection among pregnant women and its complications in their newborns during the birth in the hospitals of Dezful city, Iran, 2012-2013. Iranian Red Crescent Medical Journal, 17 (8), e26946 (2015).

44. Jazayeri, M. A. \& Irajian, G. R. Asymptomatic urinary tract infection in pregnant women.105-108. (2009).

45. Negussie, A., Worku, G. \& Beyene, E. Bacterial identification and drug susceptibility pattern of urinary tract infection in pregnant Women at Karamara Hospital Jigjiga, Eastern Ethiopia. African Journal of Bacteriology Research, 10 (2), 15-22 (2018).

46. Wabe, Y. A., Reda, D. Y., Abreham, E. T., Gobene, D. B. \& Ali, M. M. Prevalence of Asymptomatic Bacteriuria, Associated Factors and Antimicrobial Susceptibility Profile of Bacteria Among Pregnant Women Attending Saint Paul's Hospital Millennium Medical College, Addis Ababa, Ethiopia. Therapeutics and Clinical Risk Management, 16, 923 (2020).

47. Derese, B., Kedir, H., Teklemariam, Z., Weldegebreal, F. \& Balakrishnan, S. Bacterial profile of urinary tract infection and antimicrobial susceptibility pattern among pregnant women attending at Antenatal Clinic in Dil Chora Referral Hospital, Dire Dawa, Eastern Ethiopia. Therapeutics and clinical risk management, 12, 251 (2016).

48. Akpan, N. G. et al. Bacterial etiologic agents, prevalence and associated risk factors of asymptomatic bacteriuria among pregnant and non-pregnant women in primary health care centers in South-South Nigeria. International Journal of Medical and Health Research 5 (6): 66-76(2019).

49. Tessema, N. N., Ali, M. M. \& Zenebe, M. H. Bacterial associated urinary tract infection, risk factors, and drug susceptibility profile among adult people living with HIV at Haswassa University Comprehensive Specialized Hospital, Hawassa, Southern Esthiopia. Sci. Rep, 10 (1), 1-9 (2020).

50. Tadesse, S. et al. Prevalence, antimicrobial susceptibility profile and predictors of asymptomatic bacteriuria among pregnant women in Adigrat General Hospital, Northern Ethiopia. BMC research notes, 11 (1), 740 (2018).

51. Koffi, K., Aka, E., Horo, A., Britoh, A. \& Konan, J. Epidemiological, bacteriological profile and bacterial resistance of urinary tract infections at pregnant woman in prenatal consultation in African setting. International Journal of Reproduction, Contraception, Obstetrics and Gynecology, 9 (2), $461-467$ (2020).

52. Bitew, A. M., Tegene, B. M., Yeshitila, B. M., Howe, R. \& Dagnew, M. Bacterial profile, antibacterial susceptibility pattern and associated factors among women attending antenatal and post-natal health services at the University of Gondar Teaching Hospital. Northwest Ethiopia, 13, 2579-2592 (2019).

53. Tula, A., Mikru, A., Alemayehu, T. \& Dobo, B. Bacterial Profile and Antibiotic Susceptibility Pattern of Urinary Tract Infection among Pregnant Women Attending Antenatal Care at a Tertiary Care Hospital in Southern Ethiopia. Canadian Journal of Infectious Diseases and Medical Microbiology 2020, 1-9 (2020).

54. Tusuna, D. A. \& Orjino, T. A. Asymptomatic Bacteriuria Bacterial Profile and Antibiotic Susceptibility Pattern Among Pregnant Women Attending Antenatal Care (Anc) Clinic of Assosa General Hospital, Benishangul Gumuz Region, Western Ethiopia. Research square,1-9(2020).

55. Aseffa, A. et al. Bacteriological profile and antibiotic resistance pattern of urinary tract pathogens isolated at National Referral Laboratory, Ethiopian Public Health Institute. Ethiopian Journal of public health and nutrition, 2 (2), 1-6 (2020).

56. Dessalegn, D., Aseffa, A., Yitagesu, Y. \& Desta, K. Urinary Tract infection, Drug Resistance Profile and Fetal Outcomes among Pregnant women in Selected Health Facilites of Addis Ababa, Ethiopia: A cross-sectional study design. Research square,1-15(2020).

Page $13 / 14$ 
57. Nteziyaremye, J. et al. Asymptomatic bacteriuria among pregnant women attending antenatal care at Mbale Hospital, Eastern Uganda. PloS one, 15 (3), e0230523 (2020).

58. Albrich, W. C., Monnet, D. L. \& Harbarth, S. Antibiotic selection pressure and resistance in Streptococcus pneumoniae and Streptococcus pyogenes. Emerging infectious diseases, 10 (3), 514 (2004).

59. Laily, F., Lutan, D., Amelia, S., Tala, M. \& Nasution, T. Associated risk factors for urinary tract infection among pregnant women at Puskesmas Kenangan, Deli Serdang district. E\&ES 125(1), 012035(2018).

60. Shaheen, H. M., Farahat, T. M. \& Hammad, N. A. E. H. Prevalence of urinary tract infection among pregnant women and possible risk factors. Menoufia Medical Journal, 29 (4), 1055 (2016). 